NASA/TM-1999-209059

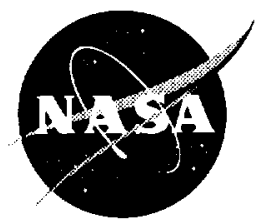

\title{
Pyrometric Gas and Surface Temperature Measurements
}

Gustave Fralick and Daniel $\mathrm{Ng}$

Glenn Research Center, Cleveland, Ohio

Prepared for the

44th International Gas Turbine and Aerospace Technical Congress sponsored by the American Society of Mechanical Engineers Indianapolis, Indiana, June 7-10, 1999

National Aeronautics and

Space Administration

Glenn Research Center 


\section{Acknowledgments}

The authors would like to thank Larry Oberle for operating the atmospheric combustor during the probe test.

This report is a preprint of a paper intended for presentation at a conference. Because of changes that may be made before formal publication, this preprint is made available with the understanding that it will not be cited or reproduced without the

permission of the author.

Available from

NASA Center for Aerospace Information

National Technical Information Service

7121 Standard Drive

5285 Port Royal Road

Hanover, MD 21076

Springfield, VA 22100

Price Code: A03

Price Code: $\mathrm{A} 03$ 


\title{
PYROMETRIC GAS AND SURFACE TEMPERATURE MEASUREMENTS
}

\author{
Gustave Fralick and Daniel $\mathrm{Ng}$ \\ National Aeronautics and Space Administration \\ Glenn Research Center \\ Cleveland, Ohio 44135
}

\begin{abstract}
A multiwavelength pyrometer possessing advantages over the one- and two-wavelength designs is described. Results of its application to surface temperature measurements of ceramics is presented. Also described is a probe suitable for gas temperature measurements to temperatures $>2600$ $K$. The design of the probe includes a multiwavelength pyrometer with fiber optic input.
\end{abstract}

\section{INTRODUCTION}

Temperature measurement of surfaces using the traditional 1-color, 2-color and other pyrometers suffers from the requirement of needing to know additional information concerning the emissivity of the measured surface and the transmissivity of the intervening optical medium. The required knowledge of these quantities ranges from knowing their absolute values at a particular wavelength to knowing their ratio at different wavelengths. This is particularly true for ceramic materials because of their complex optical properties. such as low emissivity, which varies with both temperature and wavelength. We have developed a multiwavelength pyrometer which measures the temperature of surfaces without needing to know such extensive information. This pyrometer has been used to measure the surface temperature of several materials, and has shown good agreement with surface thin film thermocouples (ref. 1). The use of the pyrometer to measure the surface temperature of two ceramics is described in this paper, as well as the use of a probe which was developed to allow this technique to be used to measure gas temperature.

\section{METHOD}

The multiwavelength pyrometer consists of a spectrometer and a computer. The computer controls the spectrometer using manufacturer's proprietary software to acquire a spectrum and performs the subsequent data analysis to determine the temperature. The temperature of the measured surface dictates the spectral region in which the spectrometer will operate. The spectral region can be from 0.5 to $2.5 \mu \mathrm{m}, 1.3$ to $4.5 \mu \mathrm{m}$ or 2 to $14.5 \mu \mathrm{m}$ depending on the particular requirements. On some occasions, depending on the equipment that is available. the actual spectral region that is selected varies slightly. The emitted/transmitted radiation is described by Planck's law of black body radiation:

$$
\begin{aligned}
& L_{\lambda}=\varepsilon_{\lambda} \tau_{\lambda} \frac{c_{1}}{\lambda^{5}} \frac{1}{\exp \left(c_{2} / \lambda T\right)-1} \\
& =\varepsilon_{\lambda} \tau_{\lambda} \frac{c_{1}}{\lambda^{5}} \exp \left(-c_{2} / \lambda T\right) \frac{1}{1-\exp \left(-c_{2} / \lambda T\right)}
\end{aligned}
$$

where $c_{1}, c_{2}$ are the radiation constants, $L_{\lambda}$ is the radiation intensity, $\varepsilon_{\lambda}$ is the emissivity, and $\tau_{\lambda}$ is the transmissivity of the optical medium between the pyrometer and the radiation source at wavelength $\lambda$.

For data analysis, Eqn. 1 is rewritten as

$$
\left(\frac{\operatorname{Ln}\left(\frac{c_{1}}{\lambda^{5}}-\frac{1}{L_{\lambda}}\right)}{c_{2} / \lambda}\right)-\frac{\operatorname{Ln}\left(1-\exp \left(-\frac{c_{2}}{\lambda T}\right)\right)}{c_{2} / \lambda}=\frac{1}{T}-\frac{\lambda}{c_{2}} \operatorname{Ln}\left(\varepsilon_{\lambda} \tau_{\lambda}\right)
$$

This is the working equation of the traditional 1-color pyrometry method which requires knowing the emissivity and or transmissivity. For the multiwavelength pyrometer, neither quantity is required to determine the temperature. Because the quantity $\left(1-\exp \left(-c_{2} / \lambda T\right)\right.$ is practically unity at short wavelengths, its logarithm is approximately zero. We observe from Eqn. 2 that plotting the quantity $y=\operatorname{Ln}\left(c_{1} /\left(\lambda^{5} L_{\lambda}\right)\right) /\left(c_{2} / \lambda\right)$ as a function of $\lambda$ would result in a straight line if $\operatorname{Ln}\left(\varepsilon_{\lambda} \tau_{\lambda}\right)$ is wavelength independent, with its slope given by $\operatorname{Ln}\left(\varepsilon_{\lambda} \tau_{\lambda}\right) / c_{2}$. The quantity $1 / y$ at each wavelength $\lambda$ is often referred to as the radiant temperature. $\tau_{\lambda}=1$, except when an optical fiber is used, and in that case its value is included in the instrument response function when it is calibrated using a black hody fumace.. The intercept of the straight line at $\lambda=0$ is $1 / T$, the reciprocal of the desired unknown temperature. After the temperature $T$ is determined, when the full expression on the left of Eqn 2 is plotted on the same graph, there is no noticeable difference. 


\section{EXPERIMENT}

(1) Zirconia $\left(\mathrm{ZrO}_{2}\right)$ Thermal Barrier Coating (TBC)

Zirconia is a ceramic material widely used to provide a thermal coating on turbine engine components for high temperature operations to achieve high efticiency and performance. The emissivity of zirconia is generally not well known. and is known to even vary with time and temperature history. The multiwavelength pyrometer recorded the spectrum of such a coating in an atmospheric jet burner (Fig. 1) with good signal to noise ratio in about 16 seconds. The spectral region from 1.5 to $4.5 \mu \mathrm{m}$ was used. The spectrum is analyzed according to Eqn. 2 and is shown in Fig. 2. From the graph's intercept, the temperature of the surface is determined. The emissivity of zirconia over most of this spectral region is constant. resulting in a very good straight line by visually fitting.

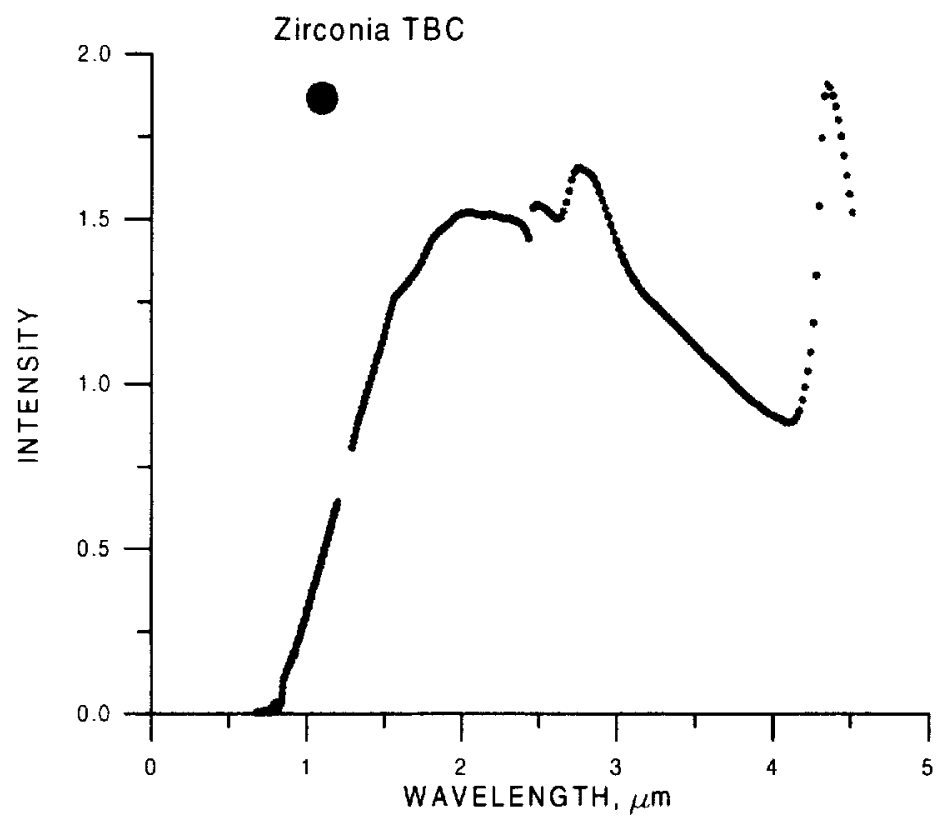

Fig. 1 Zirconia TBC spectrum

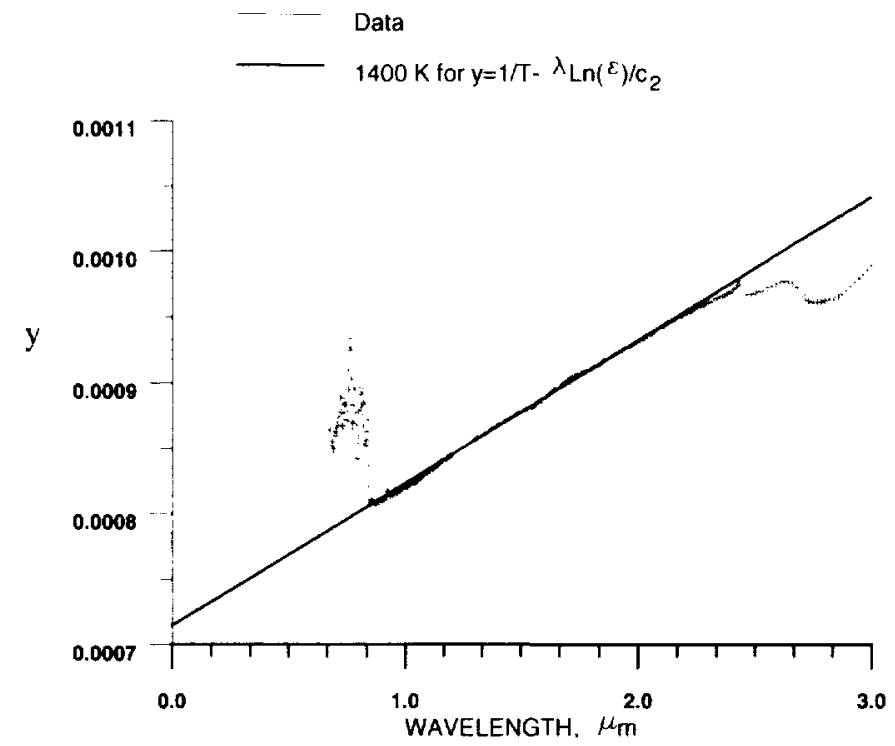

Fig. 2 Transformed zirconia TBC spectrum 


\section{(2) Beryllia (BeO)}

Beryllia is a high temperature ceramic with very low emissivity. A $6 \mathrm{~mm}$ diameter high gas temperature probe, of the type described below, was made using this material. The probe had a type $C$ thermocouple in the center. The multiwavelength pyrometer spectrometer was focused on a spot on the outside of the beryllia tubing surface to record a spectrum for temperature measurement as it was heated by a portable propane torch. The spectrum obtained is shown in Fig. 3. The transformed (according to Eqn. 2) spectrum is shown in Fig. 4. From the slope. the emissivity was determined to be 0.07 , and from the intercept the surface temperature is determined to be $1425 \mathrm{~K}$. The corresponding thermocouple reading was $1322 \mathrm{~K}$. The difference was due to poor thermal contact between the probe and the thermocouple.

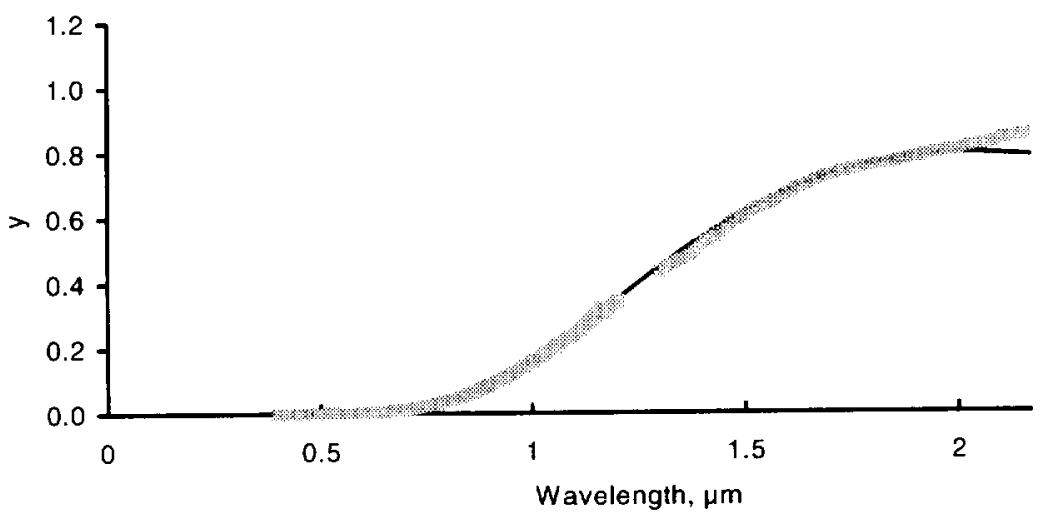

Fig. 3 BeO gas probe spectrum

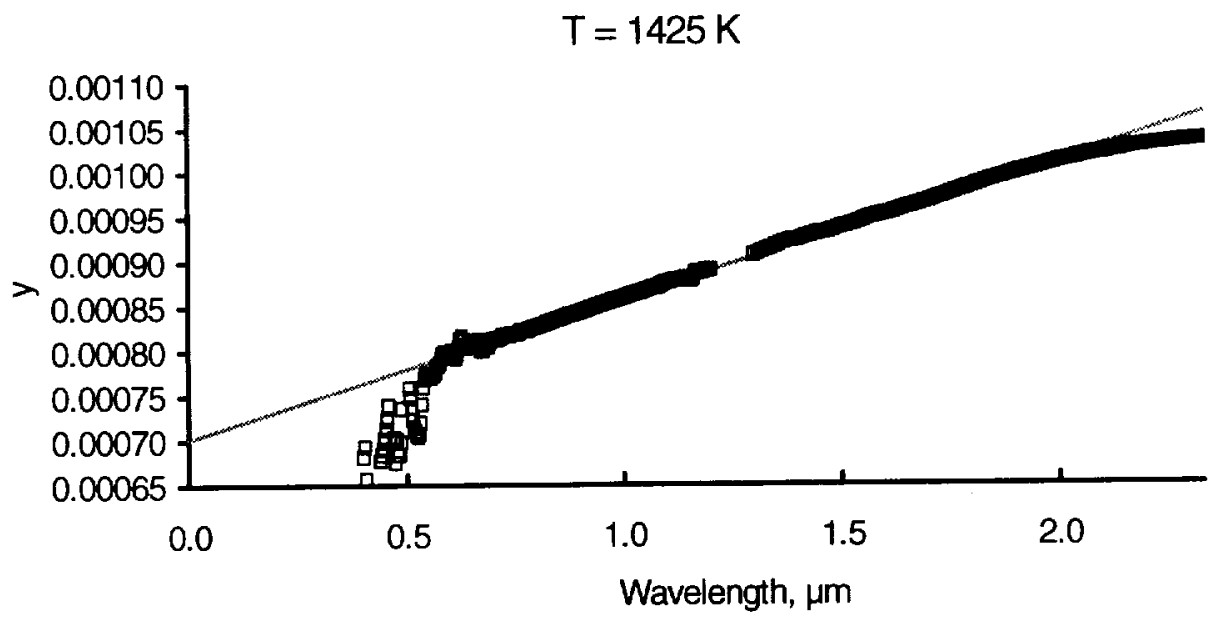

Fig. 4 BeO gas probe in propane flame 


\section{Gas Temperature Measurements}

A high gas temperature probe, designed at NASA Glenn Research Center, consists of a $\mathrm{BeO}$ sheath inserted into an inconel support section (fig. 5). Such a probe would be used to measure gas temperature in the range above about $1650 \mathrm{~K}$. as might be found in a jet engine combustor. This is beyond the range of conventional thermocouples. Although intrusive, no optical access is required; it is unlikely optical access would be available in a combustor in any case. An in-situ gas temperature measurement is useful in estimating heat loads on combustor walls. Furthermore, high gas temperatures are strongly correlated with the formation of nitrogen oxides. The overall length of the probe is $0.28 \mathrm{~m}$. the diameter of the support section is $12.7 \mathrm{~mm}$, and the sheath diameter is $8 \mathrm{~mm}$. Because of the high temperature materials employed, the probe does not need to be water cooled. As originally designed, there was a W-5\%Re/W-26\%Re (type C) thermocouple within the BeO sheath. The thermocouple is capable of measuring temperatures as high as $2589 \mathrm{~K}$. but must be in an inert atmosphere, which is the reason for the sheath. The probe has two ports. for pumping and back filling with an inert gas, or for continuously flowing a gas such as argon during a run. $\mathrm{BeO}$ was chosen as the sheath material because of its excellent high temperature electrical properties and resistance to thermal shock. Because of the necessity of keeping the thermocouple in an inert environment, and of the difficulty of relating the thermocouple temperature to the gas temperature, the authors decided to use the multi-wavelength pyrometry technique to measure the inside temperature of the $\mathrm{BeO}$ sheath. The upper temperature limit of the probe is then the useful upper limit of $\mathrm{BeO}$. which is about $2670 \mathrm{~K}$ in oxidizing atmospheres (ref. 2). The sheath acts as a black body, and because of the high thermal conductivity of beryllia. the inside and outside sheath temperatures are nearly the same. The black body signal is then transmitted from the probe to the spectrometer via an optical fiber. which is focused on the end of the sheath. The fiber itself does not need to extend into the hot region of the probe. If the heat transfer in the probe is trealed as conduction in one dimension, the gas temperature is related to the temperature of the probe tip seen by the optical fiber by the expression:

$$
T_{k}=\frac{T(L) \cosh a L-T_{b}}{\cosh a L-1}
$$

where $\mathrm{L}$ is the length of the probe, $T_{g}$ is the gas temperature, $T(L)$ is the temperature at the probe tip. $T_{b}$, is the temperature at the other end of the probe, and $a$ is a constant related to the thermal conductivity of the sheath material, the sheath thickness, and the convective heat transfer coefficient

A probe of this type was run at two different temperatures in a 3" Becon model 77-1051 atmospheric combustor. The combustor consists of a primary and secondary combustion zone. Jet fuel is injected at the entrance of the primary zone, along with sufficient air to initiate burning. Additional air is injected into the secondary zone to complete the combustion, and the flame exits through the nozzle.

The probe and spectrometer were connected by 20 meters of $3 \mathrm{~mm}$ silica fiber. The results are shown in figures $6 \&$ 7. where the indicated temperatures are $1360 \mathrm{~K}$ and 1745 $\mathrm{K}$ respectively. The estimated velocity was about 200 meters per second. The only means of comparison available was a type $S$ thermocouple inserted into the flame at the burner lip. For the two runs, it read $1300 \mathrm{~K}$ and $1580 \mathrm{~K}$ respectively. The temperature difference is explained by the fact that the probe measured the flame temperature in the center of the burner exit, which is hotter than the flame temperature at the outer radius.

Fig. 5 High Gas

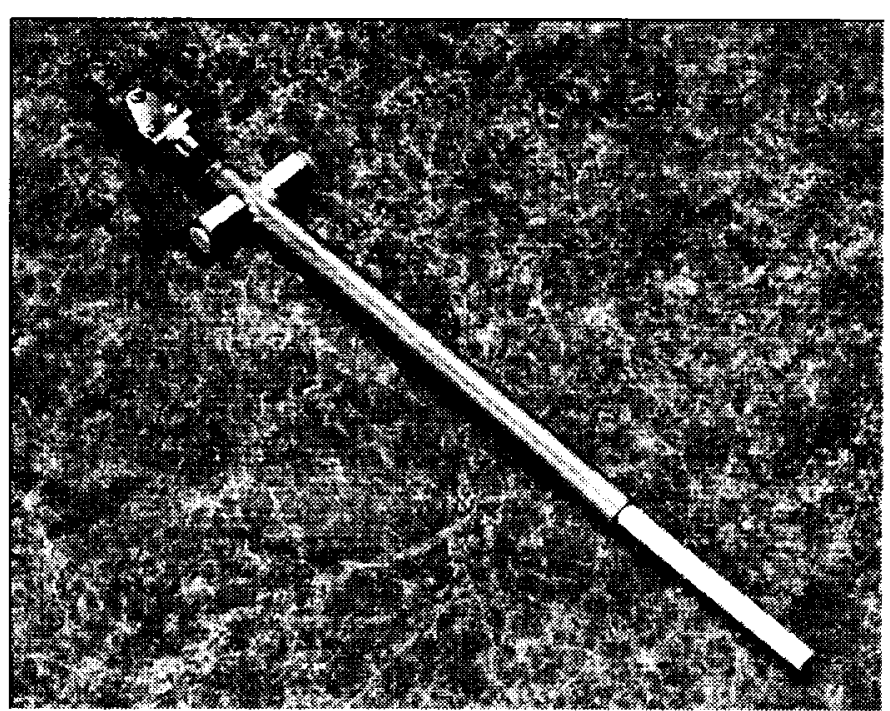

Temperature Probe 


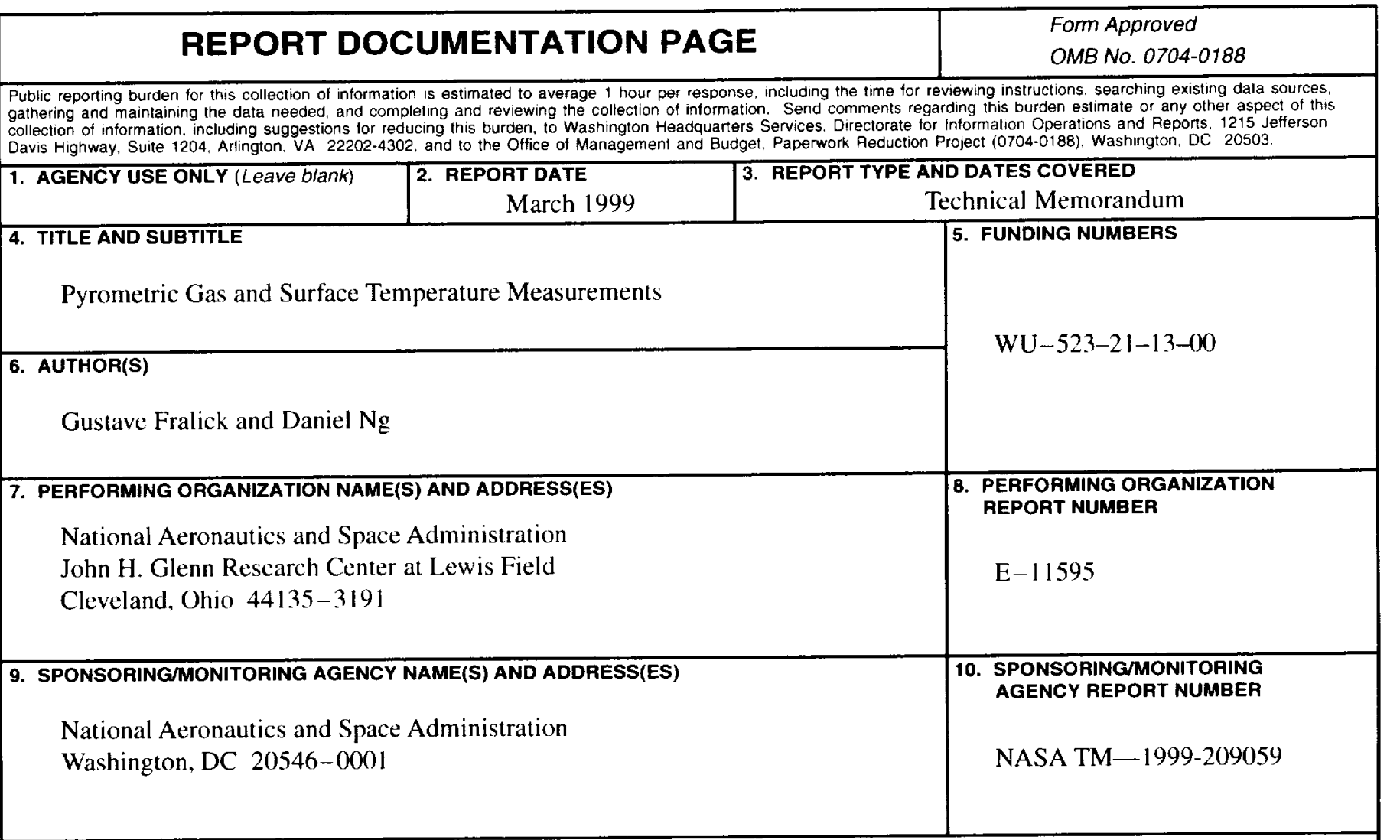

\section{SUPPLEMENTARY NOTES}

Prepared for the 44th International Gas Turbine and Aerospace Technical Congress, sponsored by the American Society of Mechanical Engineers, Indianapolis, Indiana, June 7-10, 1999. Responsible person, Gustave Fralick, organization code 5510, (216) 433-3645.

12a. DiSTRIBUTIONAVAILABILITY STATEMENT
Unclassified - Unlimited
Subject Categories: 35 and 04
This publication is available from the NASA Center for AeroSpace Information, (301) 621-0390.

A multiwavelength pyrometer possessing advantages over the one- and two-wavelength designs is described. Results of its application to surface temperature measurements of ceramics is presented. Also described is a probe suitable for gas temperature measurements to temperatures $>2600 \mathrm{~K}$. The design of the probe includes a multiwavelength pyrometer with fiber optic input.

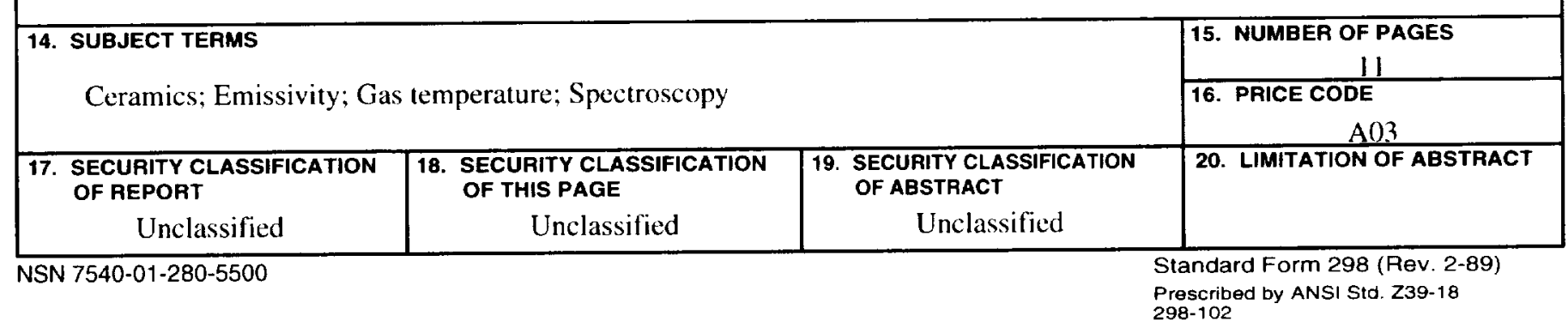


Fig. 6 Burner data, $T=1360 \mathrm{~K}$

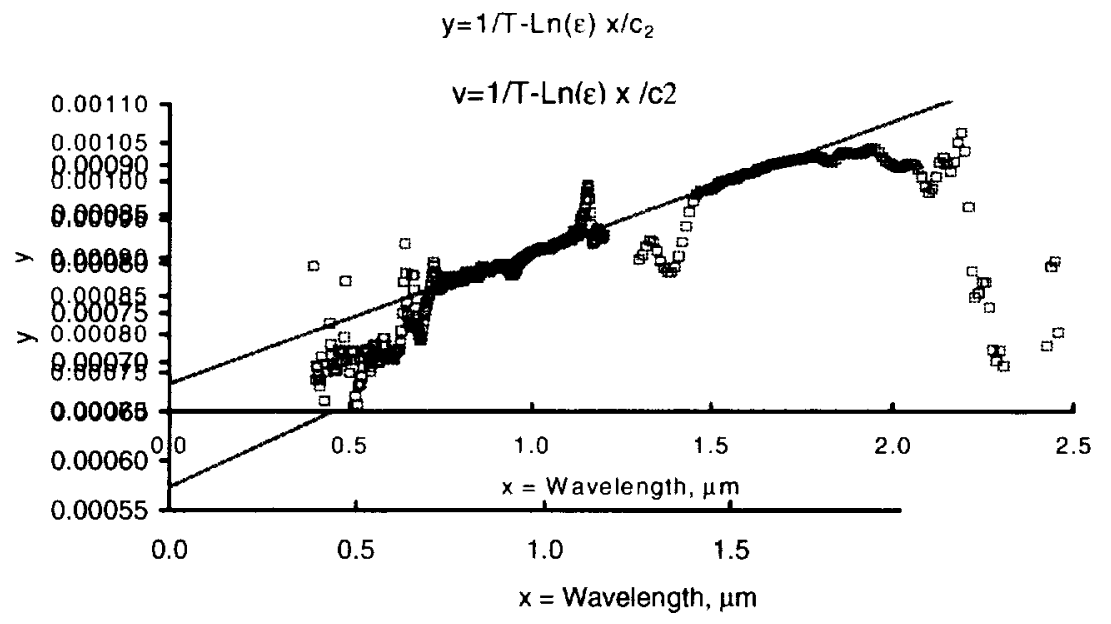

Fig. 7 Burner data, $T=1745 \mathrm{~K}$

\section{CONCLUSION}

The surface temperatures of two ceramic materials, $\mathrm{ZrO}_{2}$ and $\mathrm{BeO}$ were measured using the multiwavelength pyrometer. The multiwavelength pyrometer determines temperature by transforming the radiation spectrum over a hroad wavelength region to produce a straight line which intercepts the vertical axis. The inverse of the intercept gives the temperature. One of the surface temperature tests was in comparison with a thermocouple mounted inside the cylindrical surface whose temperature was being measured; the temperature indicated by the pyrometer was about $100 \mathrm{~K}$ higher than the thermocouple reading of $1322 \mathrm{~K}$. The pyrometer was also used in conjunction with a black body probe to measure the gas temperature at the center of an atmospheric combustor exit. In this test, the pyrometer/probe indicated a temperature higher than a thermocouple mounted at the edge of the flame. Further tests will have to be conducted to asses the accuracy of the probe.

\section{REFERENCES}

1. Ng. D., Fralick, G., Lei, J.F., Temperature Measurement of Ceramic Materials and Thermal Barrier Coatings Using a Multiwavelength Pyrometer, HITEMP Review 1997, paper 49. NASA Conference Publication 10192.

2. Campbell, I. \& Sherwood, E., Eds., High Temperature Materials and Technology, John Wiley and Sons, New York (1967) 